\title{
CONVERGENCE AND ASYMPTOTIC STABILIZATION FOR SOME DAMPED HYPERBOLIC EQUATIONS WITH NON-ISOLATED EQUILIBRIA*
}

\author{
Felipe Alvarez ${ }^{1,2}$ And Hedy Attouch ${ }^{3}$
}

\begin{abstract}
It is established convergence to a particular equilibrium for weak solutions of abstract linear equations of the second order in time associated with monotone operators with nontrivial kernel. Concerning nonlinear hyperbolic equations with monotone and conservative potentials, it is proved a general asymptotic convergence result in terms of weak and strong topologies of appropriate Hilbert spaces. It is also considered the stabilization of a particular equilibrium via the introduction of an asymptotically vanishing restoring force into the evolution equation.
\end{abstract}

Mathematics Subject Classification. 34E10, 34G05, 35B40, 35L70, 58D25.

Received June 1, 2000. Revised April 3, 2001.

\section{INTRODUCTION}

Classical methods to establish the asymptotic convergence of trajectories of dissipative dynamical systems assume isolated equilibrium points (local uniqueness). However, in many interesting cases the set of all equilibria is a continuum of stationary solutions so that local uniqueness does not hold. The aim of this article is to show that in the case of some infinite-dimensional second-order in time evolution equations, global monotonicity conditions allow one to overcome the lack of uniqueness of stationary solutions in order to establish asymptotic convergence. This work is motivated by a recent result of the first author concerning the asymptotic convergence of the trajectories generated by a gradient equation of the type

$$
\frac{\mathrm{d}^{2} u}{\mathrm{~d} t^{2}}(t)+\alpha \frac{\mathrm{d} u}{\mathrm{~d} t}(t)+\nabla \Phi(u(t))=0, t>0,
$$

where $\alpha>0$ is a real parameter, $\Phi: H \rightarrow \mathbb{R}$ is assumed to be continuously differentiable and convex, and $H$ is a real Hilbert space. When the set of minimizers of $\Phi$ is nonempty, it is proved in [1] that every solution trajectory of (1) converges to a minimizer of $\Phi$ for the weak topology of $H$ (see [4] for others results in this connection). Observe that the convexity assumption on $\Phi$ amounts to the monotonicity of $\nabla \Phi$, that is, $\forall x$,

Keywords and phrases: Second-order in time equation, linear damping, dissipative hyperbolic equation, weak solution, asymptotic behavior, stabilization, weak convergence, Hilbert space.

* This work was partially supported by the French-Chilean Research Cooperation Program ECOS-CONICYT. The first author was also supported by grants FONDECYT 1990884 and FONDAP in Applied Mathematics.

${ }^{1}$ Depto. Ingeniería Matemática, Universidad de Chile, Casilla 170/3, Correo 3, Santiago, Chile; e-mail: falvarez@dim.uchile.cl

2 Centro de Modelamiento Matemático (CNRS UMR 2071), Universidad de Chile, Blanco Encalada 2120, Santiago, Chile.

${ }^{3}$ Laboratoire ACSIOM-CNRS FRE 2311, Département de Mathématiques, Université de Montpellier II, Place Eugène Bataillon, 34095 Montpellier Cedex 05, France; e-mail: attouch@math.univ-montp2.fr

(c) EDP Sciences, SMAI 2001 
$y \in H,(\nabla \Phi(x)-\nabla \Phi(y), x-y) \geq 0$. Of course, the regularity condition on $\Phi$ is too restrictive in view of eventual applications to PDE. Nevertheless, it turns out that a more careful inspection of the proofs from [1] permits us to extend the key arguments to cover actual hyperbolic problems.

This paper is organized as follows. We begin by considering in Section 2 the case of linear abstract equations of the type

$$
\frac{\mathrm{d}^{2} u}{\mathrm{~d} t^{2}}(t)+\alpha \frac{\mathrm{d} u}{\mathrm{~d} t}(t)+A u(t)=0, t>0,
$$

where $\alpha>0, V$ and $H$ are real Hilbert spaces such that $V \subset H \subset V^{\prime}$ with dense and continuous injections ${ }^{4}$, $u(0)=u_{0} \in V, \frac{\mathrm{d} u}{\mathrm{~d} t}(0)=v_{0} \in H$, and $A: V \rightarrow V^{\prime}$ is a symmetric, "weakly" coercive, linear monotone operator. It is shown that the weak solution $u(t)$ converges in $V$, as $t \rightarrow \infty$, toward the projection in $H$ of $u_{0}+\frac{1}{\alpha} v_{0}$ onto the kernel of $A$. Then we deal with the stabilization of the particular stationary solution $u=0$ by introducing into the equation an asymptotically vanishing restoring force of the type $\varepsilon(t) u$, with $\varepsilon(t)>0$ and $\varepsilon(t) \rightarrow 0$ as $t \rightarrow \infty$. Concerning the nonlinear case, an abstract result is established in Section 3 for an equation of the form

$$
\frac{\mathrm{d}^{2} u}{\mathrm{~d} t^{2}}(t)+\alpha \frac{\mathrm{d} u}{\mathrm{~d} t}(t)+A u(t)+f(u(t))=0, t>0,
$$

where $f: V \rightarrow H$ is continuous, monotone and conservative. In contrast with the linear case, no characterization of the limit solution is given and the convergence toward an equilibrium is proved only for the weak topology of $V$; although strong convergence is an open problem in the general case, it does hold under compactness or symmetry conditions. A nonlinear stabilization result is given, whose proof is briefly outlined. Finally, the abstract convergence result is illustrated with some examples in Section 4.

Results and techniques similar in spirit to those presented here are well-known in the theory of first-order in time evolution equations associated with monotone operators ( $c f$. Brezis [6] and Bruck [7]; see also [2, 10,12, $20])$. With regard to damped second-order in time evolution equations with non-isolated equilibria, asymptotic convergence results were obtained by Haraux [15] and Zuazua [23] using different tools; in the nonlinear case, they assumed in addition that the kernel of $A$ is one-dimensional. On the other hand, more recent general convergence results by Hale and Raugel [14] and Brunovsky and Polacik [8] ensure in this context asymptotic convergence without requiring monotonicity of $f$ but under the additional assumption that the kernel of $A$ is one-dimensional and finite dimensional respectively. This type of result is well-known in finite dimensional dynamical system theory under hyperbolic assumptions on the manifold of stationary solutions; see for instance [5]. The finite dimension hypothesis on the kernel of $A$ is unnecessarily in our approach and, moreover, we only use elementary functional and energy methods. Finally, it is worth pointing out that when the nonlinearity is supposed to be analytic instead of monotone, it is possible to establish the asymptotic convergence of the trajectory by different methods; $c f$. Simon [21] for parabolic problems and the recent works by Haraux and Jendoubi [17, 18] for hyperbolic ones (under Dirichlet boundary conditions).

\section{LiNEAR EQUATIONS}

\subsection{Asymptotic convergence to an equilibrium}

Let us begin with a quite simple situation. Given a regular, bounded and connected domain $\Omega \subset \mathbb{R}^{N}$, and a real parameter $\alpha>0$, let $u(x, t): \Omega \times[0, \infty[\rightarrow \mathbb{R}$ be a weak solution of the linear damped wave equation

$$
\left.u_{t t}+\alpha u_{t}-\Delta u=0 \text { in } \Omega \times\right] 0, \infty[,
$$

under homogeneous Neumann boundary conditions

$$
\left.\frac{\partial u}{\partial \mathbf{n}}=0 \text { on } \partial \Omega \times\right] 0, \infty[.
$$

${ }^{4} V^{\prime}$ stands for the dual of $V$. 
The corresponding set of stationary solutions consists of all constant functions on $\Omega$. If we assume that $u(x, 0)=$ $u_{0}(x)$ and $u_{t}(x, 0)=v_{0}(x)$ with $u_{0} \in H^{1}(\Omega)$ and $v_{0} \in L^{2}(\Omega)$, then it is simple to verify that the Fourier decomposition of solutions on the basis of the eigenfunctions of the Neumann Laplacian yields $\left(u(\cdot, t), u_{t}(\cdot, t)\right) \rightarrow$ $\left(u_{\infty}, 0\right)$ strongly in $H^{1}(\Omega) \times L^{2}(\Omega)$ as $t \rightarrow \infty$, with $u_{\infty}$ being the constant function given by

$$
u_{\infty} \equiv \frac{1}{|\Omega|} \int_{\Omega}\left[u_{0}(x)+\frac{1}{\alpha} v_{0}(x)\right] \mathrm{d} x,
$$

where $|\Omega|$ is the Lebesgue measure of $\Omega$.

In order to extend this type of convergence result to more general situations, let us consider two real Hilbert spaces $V$ and $H$ with $V \subset H$. The scalar product and the norm on $H$ and $V$ are denoted by $(\cdot, \cdot),|\cdot|$ and $((\cdot, \cdot)),\|\cdot\|$ respectively. It is supposed that $V$ is dense in $H$ with the injection being continuous, and $H$ is identified with its dual $H^{\prime}$ and with a dense subspace of the dual $V^{\prime}$ of $V$. Thus $V \subset H \subset V^{\prime}, H$ being dense in $V^{\prime}$ with continuous injection. We denote by $\langle\cdot, \cdot\rangle_{V^{\prime}, V}$ the duality product between $V^{\prime}$ and $V$. Recall that with the above identifications, $\langle u, v\rangle_{V^{\prime}, V}=(u, v)$ whenever $u \in H$ and $v \in V$. Let $a: V \times V \rightarrow \mathbb{R}$ be a continuous bilinear form satisfying:

$\left(\mathrm{h}_{1}\right) a(\cdot, \cdot)$ is symmetric: $\forall u, v \in V, a(u, v)=a(v, u)$;

$\left(\mathrm{h}_{2}\right)$ There exist $\lambda \geq 0$ and $\mu>0$ such that $\forall u \in V, a(u, u)+\lambda|u|^{2} \geq \mu\|u\|^{2}$;

$\left(\mathrm{h}_{3}\right) a(\cdot, \cdot)$ is monotone: $\forall u \in V, a(u, u) \geq 0$.

Associating with $a(\cdot, \cdot)$ the continuous operator $A: V \rightarrow V^{\prime}$ given by $\langle A u, v\rangle_{V^{\prime}, V}:=a(u, v)$, we consider the following abstract linear evolution equation of the second-order in time:

$$
\frac{\mathrm{d}^{2} u}{\mathrm{~d} t^{2}}(t)+\alpha \frac{\mathrm{d} u}{\mathrm{~d} t}(t)+A u(t)=0, t>0
$$

where $\alpha>0$ is a real parameter. A weak solution of this problem is a function $u(\cdot) \in C\left(\left[0, \infty[; V) \cap C^{1}([0, \infty[; H)\right.\right.$ verifying for every $v \in V$

$$
\frac{\mathrm{d}}{\mathrm{d} t}\left(\frac{\mathrm{d} u}{\mathrm{~d} t}(\cdot), v\right)+\alpha\left(\frac{\mathrm{d} u}{\mathrm{~d} t}(\cdot), v\right)+a(u, v)=0,
$$

in the scalar distribution sense. The existence and uniqueness under initial conditions of such a solution $u(t)$ is well-known in infinite-dimensional dynamical systems theory; see [11] (Chap. XVIII). Moreover, the following energy equation holds:

$$
\frac{\mathrm{d} E}{\mathrm{~d} t}+\alpha\left|\frac{\mathrm{d} u}{\mathrm{~d} t}\right|^{2}=0
$$

where

$$
E(t):=\frac{1}{2}\left|\frac{\mathrm{d} u}{\mathrm{~d} t}(t)\right|^{2}+\frac{1}{2} a(u(t), u(t)) .
$$

Theorem 2.1. Under $\left(\mathrm{h}_{1}-\mathrm{h}_{3}\right)$, for every $\alpha>0, u_{0} \in V$ and $v_{0} \in H$, the unique solution $u(t)$ of the initial-value problem

$$
\left\{\begin{array}{l}
\frac{\mathrm{d}^{2} u}{\mathrm{~d} t^{2}}(t)+\alpha \frac{\mathrm{d} u}{\mathrm{~d} t}(t)+A u(t)=0, t>0, \\
u(0)=u_{0}, \quad \frac{\mathrm{d} u}{\mathrm{~d} t}(0)=v_{0},
\end{array}\right.
$$

satisfies $\frac{\mathrm{d} u}{\mathrm{~d} t} \in L^{2}(0, \infty ; H)$, there exists $C \geq 0$ such that $E(t) \leq C / t$, and $\left(u(t), \frac{\mathrm{d} u}{\mathrm{~d} t}(t)\right) \rightarrow\left(u_{\infty}, 0\right)$ strongly in $V \times H$ as $t \rightarrow \infty$, with

$$
u_{\infty}=\operatorname{Proj}_{\text {ker } A}\left(u_{0}+\frac{1}{\alpha} v_{0}\right) .
$$


Here, $\operatorname{Proj}_{\mathrm{ker} A}(z)$ stands for the orthogonal projection of $z \in H$ onto $\operatorname{ker} A:=\{v \in V: A v=0\}$, relative to the scalar product $(\cdot, \cdot)$ on $H$.

Proof. From (3), it follows that

$$
E(t)+\alpha \int_{0}^{t}\left|\frac{\mathrm{d} u}{\mathrm{~d} t}(\tau)\right|^{2} \mathrm{~d} \tau=E(0)
$$

In particular,

$$
\int_{0}^{\infty}\left|\frac{\mathrm{d} u}{\mathrm{~d} t}(\tau)\right|^{2} \mathrm{~d} \tau \leq \frac{1}{2 \alpha}\left[\left|v_{0}\right|^{2}+a\left(u_{0}, u_{0}\right)\right]
$$

Thus $\frac{\mathrm{d} u}{\mathrm{~d} t} \in L^{2}(0, \infty ; H)$. Define now the auxiliary real-valued function $\varphi(t):=\frac{1}{2}|u(t)|^{2}$. We have $\varphi \in$ $C^{1}\left(0, \infty ; \mathbb{R}_{+}\right)$with $\dot{\varphi}(t)=\left(u(t), \frac{\mathrm{d} u}{\mathrm{~d} t}(t)\right)$. Since $\frac{\mathrm{d}^{2} u}{\mathrm{~d} t^{2}}(t)+A u(t) \in H$, a standard argument yields

$$
\frac{\mathrm{d}}{\mathrm{d} t}[\dot{\varphi}(t)]=\left(\frac{\mathrm{d}^{2} u}{\mathrm{~d} t^{2}}(t)+A u(t), u(t)\right)-a(u(t), u(t))+\left|\frac{\mathrm{d} u}{\mathrm{~d} t}(t)\right|^{2}
$$

which holds in the scalar distribution sense on $] 0, T$ [ for every $T>0$ (see, for instance [22], Chap. II, Lem. 4.1). In this case, the latter amounts to $\ddot{\varphi}(t)=-\alpha \dot{\varphi}(t)-a(u(t), u(t))+\left|\frac{\mathrm{d} u}{\mathrm{~d} t}(t)\right|^{2}$ in the classical sense. This equation may be rewritten

$$
\ddot{\varphi}(t)+\alpha \dot{\varphi}(t)+2 E(t)=2\left|\frac{\mathrm{d} u}{\mathrm{~d} t}(t)\right|^{2}
$$

Using that $E(t)$ is non-increasing, a simple integration procedure shows that for every $\theta \in[0, t]$

$$
\dot{\varphi}(\theta)+\frac{2}{\alpha}\left(1-\mathrm{e}^{-\alpha \theta}\right) E(t) \leq \mathrm{e}^{-\alpha \theta} \dot{\varphi}(0)+2 \int_{0}^{\theta} \mathrm{e}^{-\alpha(\theta-\tau)}\left|\frac{\mathrm{d} u}{\mathrm{~d} t}(\tau)\right|^{2} \mathrm{~d} \tau .
$$

Hence

$$
\varphi(t)+\frac{2}{\alpha^{2}}\left(\mathrm{e}^{-\alpha t}-1+\alpha t\right) E(t) \leq \varphi(0)+\frac{1}{\alpha}\left(1-\mathrm{e}^{-\alpha t}\right) \dot{\varphi}(0)+2 h(t)
$$

where

$$
h(t):=\int_{0}^{t} \int_{0}^{\theta} \mathrm{e}^{-\alpha(\theta-\tau)}\left|\frac{\mathrm{d} u}{\mathrm{~d} t}(\tau)\right|^{2} \mathrm{~d} \tau \mathrm{d} \theta
$$

We conclude that

$$
t E(t) \leq \frac{\alpha}{2} \varphi(0)+\frac{1}{2}\left(1-\mathrm{e}^{-\alpha t}\right)\left[\dot{\varphi}(0)+\frac{2}{\alpha} E(0)\right]+\alpha h(t),
$$

which holds for every $t \geq 0$. Furthermore, by Fubini's theorem

$$
h(t)=\int_{0}^{t} \int_{\theta}^{t} \mathrm{e}^{-\alpha(\theta-\tau)}\left|\frac{\mathrm{d} u}{\mathrm{~d} t}(\tau)\right|^{2} \mathrm{~d} \tau \mathrm{d} \theta=\frac{1}{\alpha} \int_{0}^{t}\left(1-\mathrm{e}^{-\alpha(t-\tau)}\right)\left|\frac{\mathrm{d} u}{\mathrm{~d} t}(\tau)\right|^{2} \mathrm{~d} \tau \leq \frac{1}{\alpha} \int_{0}^{\infty}\left|\frac{\mathrm{d} u}{\mathrm{~d} t}(\tau)\right|^{2} \mathrm{~d} \tau<\infty .
$$

Therefore $h(t)$ is bounded and, as a consequence, there exists a constant $C \geq 0$ such that $E(t) \leq C / t$. Therefore $\lim _{t \rightarrow \infty}\left|\frac{\mathrm{d} u}{\mathrm{~d} t}(t)\right|=\lim _{t \rightarrow \infty} a(u(t), u(t))=0$. Moreover $\{\varphi(t)\}_{t>0}$ is bounded so that the trajectory $\{u(t): t \rightarrow \infty\}$ is bounded in $H$. By $\left(\mathrm{h}_{2}\right),\{u(t): t \rightarrow \infty\}$ is bounded in $V$. 
To establish the convergence of $u(t)$ in $V$, let us consider a sequence $t_{k} \rightarrow \infty$ such that $u\left(t_{k}\right) \rightarrow \widehat{u}$ weakly in $V$ for some $\widehat{u} \in V$. By weak lower semi-continuity of $v \in V \rightarrow a(v, v)$, we have

$$
a(\widehat{u}, \widehat{u}) \leq \liminf _{k \rightarrow \infty} a\left(u\left(t_{k}\right), u\left(t_{k}\right)\right)=\lim _{t \rightarrow \infty} a(u(t), u(t))=0
$$

It follows easily from $a(\widehat{u}, \widehat{u})=0$ that for every $v \in V, a(\widehat{u}, v)=0$, hence $A \widehat{u}=0$. When ker $A$ is a singleton, we conclude that $\{u(t): t \rightarrow \infty\}$ admits a unique cluster point for the weak topology in $V$, hence that $u(t) \rightarrow u_{\infty}$ weakly in $V$ as $t \rightarrow \infty$, where $u_{\infty}$ is the unique solution of $A u_{\infty}=0$. In the general case, observe that for every $v \in \operatorname{ker} A$ we have that

and consequently

$$
\frac{\mathrm{d}}{\mathrm{d} t}\left(\frac{\mathrm{d} u}{\mathrm{~d} t}, v\right)+\alpha\left(\frac{\mathrm{d} u}{\mathrm{~d} t}, v\right)=0
$$

$$
(u(t), v)=\left(u_{0}+\frac{1}{\alpha} v_{0}, v\right)-\frac{1}{\alpha}\left(\frac{\mathrm{d} u}{\mathrm{~d} t}(t), v\right) .
$$

Hence $\left(\widehat{u}-u_{0}-\frac{1}{\alpha} v_{0}, v\right)=0$. Here, we have used that $u\left(t_{k}\right) \rightarrow \widehat{u}$ weakly in $H$, which is true because the injection from $V$ into $H$ is continuous. Thus, $u_{\infty}:=\operatorname{Proj}_{\text {ker } A}\left(u_{0}+\frac{1}{\alpha} v_{0}\right)$ is the unique cluster point of $\{u(t): t \rightarrow \infty\}$ for the weak topology in $V$, and so $u(t) \rightarrow u_{\infty}$ weakly in $V$ as $t \rightarrow \infty$. It remains to prove that $u(t) \rightarrow u_{\infty}$ strongly in $V$. By $\left(\mathrm{h}_{1}, \mathrm{~h}_{2}\right)$ and the fact that $a\left(u_{\infty}, u(t)\right)=0$, we have

$$
\mu\left\|u(t)-u_{\infty}\right\|^{2} \leq \lambda\left|u(t)-u_{\infty}\right|^{2}+a\left(u(t)-u_{\infty}, u(t)-u_{\infty}\right)=\lambda\left|u(t)-u_{\infty}\right|^{2}+a(u(t), u(t)),
$$

which gives

$$
\limsup _{t \rightarrow \infty}\left\|u(t)-u_{\infty}\right\| \leq \sqrt{\lambda / \mu} \limsup _{t \rightarrow \infty}\left|u(t)-u_{\infty}\right|
$$

To finish the proof, it suffices to show that $u(t) \rightarrow u_{\infty}$ strongly in $H$ as $t \rightarrow \infty$. This is immediate under ( $\left.\mathrm{h}_{2}\right)$ when the injection from $V$ into $H$ is compact. When the injection is only continuous, it is possible to adapt to this situation some arguments of [7], where a class of first-order in time equations is treated. Fix $t_{0}>0$ and define $q:\left[0, t_{0}\right] \rightarrow \mathbb{R}$ by

$$
q(t):=|u(t)|^{2}-\left|u\left(t_{0}\right)\right|^{2}-\frac{1}{2}\left|u(t)-u\left(t_{0}\right)\right|^{2}
$$

Then $\left.\ddot{q}(t)+\alpha \dot{q}(t)=-a(u(t)), u(t)+u\left(t_{0}\right)\right)+\left|\frac{\mathrm{d} u}{\mathrm{~d} t}(t)\right|^{2}$. Since $E(t)$ is non-increasing, we deduce that for every $t \in\left[0, t_{0}\right]$

$$
\begin{aligned}
\frac{1}{2}\left|\frac{\mathrm{d} u}{\mathrm{~d} t}(t)\right|^{2} & \geq \frac{1}{2}\left|\frac{\mathrm{d} u}{\mathrm{~d} t}\left(t_{0}\right)\right|^{2}+\frac{1}{2}\left[a\left(u\left(t_{0}\right), u\left(t_{0}\right)\right)-a(u(t), u(t))\right] \\
& =\frac{1}{2}\left|\frac{\mathrm{d} u}{\mathrm{~d} t}\left(t_{0}\right)\right|^{2}+\frac{1}{2}\left[a\left(u\left(t_{0}\right), u(t)+u\left(t_{0}\right)\right)-a\left(u(t), u(t)+u\left(t_{0}\right)\right)\right] \\
& \geq \frac{1}{2}\left|\frac{\mathrm{d} u}{\mathrm{~d} t}\left(t_{0}\right)\right|^{2}-a\left(u(t), u(t)+u\left(t_{0}\right)\right)
\end{aligned}
$$

where we have used the inequality $a(v, u+v) \geq-a(u, u+v)$. Consequently, $\ddot{q}(t)+\alpha \dot{q}(t) \leq \frac{3}{2}\left|\frac{\mathrm{d} u}{\mathrm{~d} t}(t)\right|^{2}$ for every $t \in\left[0, t_{0}\right]$. The standard integration procedure yields

$$
q\left(t_{0}\right)-q(t) \leq \frac{\dot{q}(0)}{\alpha}\left(\mathrm{e}^{-\alpha t}-\mathrm{e}^{-\alpha t_{0}}\right)+\frac{3}{2} \int_{t}^{t_{0}} \int_{0}^{\theta} \mathrm{e}^{-\alpha(\theta-\tau)}\left|\frac{\mathrm{d} u}{\mathrm{~d} t}(\tau)\right|^{2} \mathrm{~d} \tau \mathrm{d} \theta
$$

Therefore, for all $t \in\left[0, t_{0}\right]$ we have

$$
\frac{1}{2}\left|u(t)-u\left(t_{0}\right)\right|^{2} \leq|u(t)|^{2}-\left|u\left(t_{0}\right)\right|^{2}+\frac{\dot{q}(0)}{\alpha}\left(\mathrm{e}^{-\alpha t}-\mathrm{e}^{-\alpha t_{0}}\right)+\frac{3}{2}\left[h\left(t_{0}\right)-h(t)\right],
$$


where $h$ is given by (6). Since $\frac{\mathrm{d} u}{\mathrm{~d} t} \in L^{2}(0, \infty ; H)$, by Fubini's theorem $h(t)$ converges as $t \rightarrow \infty$. On the other hand, from (4) it follows that

$$
\ddot{\varphi}(t)+\alpha \dot{\varphi}(t) \leq 2\left|\frac{\mathrm{d} u}{\mathrm{~d} t}(t)\right|^{2} .
$$

Lemma 2.2. Let $\varphi \in C^{1}([0, \infty[; \mathbb{R})$ be bounded from below and assume that $\dot{\varphi}$ is absolutely continuous. If there exist $\alpha>0$ and $p \in L^{1}(0, \infty ; \mathbb{R})$ such that $\ddot{\varphi}(t)+\alpha \dot{\varphi}(t) \leq p(t)$ for a.e. $t>0$, then $\varphi(t)$ converges as $t \rightarrow \infty$.

Proof. From the inequality $\ddot{\varphi}(t)+\alpha \dot{\varphi}(t) \leq p(t)$, it follows that

$$
\dot{\varphi}(t) \leq \mathrm{e}^{-\alpha t} \dot{\varphi}(0)+\int_{0}^{t} \mathrm{e}^{-\alpha(t-\tau)}|p(\tau)| \mathrm{d} \tau
$$

Thus

$$
[\dot{\varphi}(t)]_{+} \leq \mathrm{e}^{-\alpha t}[\dot{\varphi}(0)]_{+}+q(t)
$$

where $[x]_{+}=\max \{x, 0\}$ and $q(t):=\int_{0}^{t} \mathrm{e}^{-\alpha(t-\tau)}|p(\tau)| \mathrm{d} \tau$. Fubini's theorem gives $q \in L^{1}(0, \infty ; \mathbb{R})$ and therefore $[\dot{\varphi}]_{+} \in L^{1}(0, \infty ; \mathbb{R})$. Setting $w(t):=\varphi(t)-\int_{0}^{t}[\dot{\varphi}(\tau)]_{+} \mathrm{d} \tau$, we have that $w(t)$ is bounded from below. But $w(t)$ is non-increasing because $\dot{w}(t)=\dot{\varphi}(t)-[\dot{\varphi}(t)]_{+} \leq 0$. Hence $w(t)$ converges as $t \rightarrow \infty$, and consequently $\varphi(t)$ is convergent as well.

Let us return to the proof of Theorem 2.1. We claim that $\lim _{t \rightarrow \infty}|u(t)|$ exists. Indeed, we can apply Lemma 2.2 with $p(t)=2\left|\frac{\mathrm{d} u}{\mathrm{~d} t}(t)\right|^{2}$ to conclude that $\varphi(t)=\frac{1}{2}|u(t)|^{2}$ converges as $t \rightarrow \infty$. We infer from $(7)$ that $\{u(t): t \rightarrow \infty\}$ is a Cauchy generalized sequence in $H$. This completes the proof of the theorem.

Remark 2.3. A similar abstract equation is treated in [23] (Sect. 4), where, instead of $\left(\mathrm{h}_{2}\right)$, it is supposed that

$$
\exists \eta>0: \forall u \in V, a(u, u) \geq \eta\left\|u-\operatorname{Proj}_{\operatorname{ker} A}(u)\right\|^{2} .
$$

In fact, if (8) holds then there exist two constants $k, \delta>0$ such that

$$
\left\|u(t)-\operatorname{Proj}_{\text {ker } A}(u(t))\right\|+\left|\frac{\mathrm{d} u}{\mathrm{~d} t}(t)\right| \leq k \mathrm{e}^{-\delta t}
$$

In particular, $\frac{\mathrm{d} u}{\mathrm{~d} t} \in L^{1}(0, \infty ; H)$ and we deduce that $u(t)$ converges strongly in $H$ as $t \rightarrow \infty$ (see [23], Rem. 4.4). On the other hand, Theorem 2.1 ensures strong convergence in $V$ without assuming a priori that the trajectory $\{u(t): t \rightarrow \infty\}$ is precompact in $V$, and, moreover, the limit solution $u_{\infty}$ is completely characterized. Furthermore, in the convergence analysis we use $\left(\mathrm{h}_{2}\right)$ only to establish boundedness and convergence in $V$. Thus, if we drop assumption $\left(\mathrm{h}_{2}\right)$ then we can deduce that every weak solution of $(2)$ converges as $t \rightarrow \infty$ for the strong topology in $H$.

\subsection{Stabilization of a particular stationary solution}

By appropriately adjusting the initial conditions, one could let the trajectory asymptotically reach any particular target equilibrium. Nevertheless, in many situations it is not possible to have an accurate control of the initial state. An alternative approach consists in adjusting the forces acting on the system. For instance, if we introduce into the differential equation a restoring force of the type $k u$ with $k>0$, then it is trivial to show that the equilibrium $u=0$ is globally asymptotically stable, i.e. every trajectory $u(t)$ converges to 0 as $t \rightarrow \infty$. This is not surprising because when replacing $A u$ by $A u+k u, u=0$ is the unique stationary solution of the corresponding evolution equation. More interesting is the case where $A u$ is replaced by $A u+\varepsilon(t) u$ with $\varepsilon(t)>0$ and $\varepsilon(t) \rightarrow 0$ as $t \rightarrow \infty$. Since in this situation the restoring force asymptotically vanishes as $t \rightarrow \infty$, convergence towards 0 is more delicate. 
Theorem 2.4. Assume $\left(\mathrm{h}_{1}-\mathrm{h}_{3}\right)$ and let $u \in C\left(\left[0, \infty[; V) \cap C^{1}([0, \infty[; H)\right.\right.$ be a solution of the following nonautonomous evolution problem:

$$
\frac{\mathrm{d}^{2} u}{\mathrm{~d} t^{2}}(t)+\alpha \frac{\mathrm{d} u}{\mathrm{~d} t}(t)+A u(t)+\varepsilon(t) u(t)=0, t>0
$$

where $\alpha>0$ and $\varepsilon:[0, \infty[\rightarrow[0, \infty[$ is a given differentiable function such that for all $t \geq 0, \dot{\varepsilon}(t) \leq 0$. Then $\frac{\mathrm{d} u}{\mathrm{~d} t} \in L^{2}(0, \infty ; H)$, there exists $C \geq 0$ such that $E_{\varepsilon(t)}(t) \leq C / t$ where

$$
E_{\varepsilon(t)}(t):=\frac{1}{2}\left|\frac{\mathrm{d} u}{\mathrm{~d} t}(t)\right|^{2}+a(u(t), u(t))+\frac{\varepsilon(t)}{2}|u(t)|^{2},
$$

and if we assume that

$$
\int_{0}^{\infty} \varepsilon(\tau) \mathrm{d} \tau=\infty
$$

then $\left(u(t), \frac{\mathrm{d} u}{\mathrm{~d} t}(t)\right) \rightarrow(0,0)$ strongly in $V \times H$ as $t \rightarrow \infty$.

Proof. It is easy to verify that in this case

$$
\frac{\mathrm{d}}{\mathrm{d} t}\left[E_{\varepsilon(t)}(t)\right]=-\alpha\left|\frac{\mathrm{d} u}{\mathrm{~d} t}(t)\right|^{2}+\frac{\dot{\varepsilon}(t)}{2}|u(t)|^{2} .
$$

Since $\dot{\varepsilon}(t) \leq 0$, we deduce that $E_{\varepsilon(t)}(t)$ is non-increasing and

$$
E_{\varepsilon(t)}(t)+\alpha \int_{0}^{t}\left|\frac{\mathrm{d} u}{\mathrm{~d} t}(\tau)\right|^{2} \mathrm{~d} \tau=E_{\varepsilon_{0}}(0)+\frac{1}{2} \int_{0}^{t} \dot{\varepsilon}(\tau)|u(\tau)|^{2} \mathrm{~d} \tau \leq E_{\varepsilon_{0}}(0) .
$$

Consequently,

$$
\int_{0}^{\infty}\left|\frac{\mathrm{d} u}{\mathrm{~d} t}(\tau)\right|^{2} \mathrm{~d} \tau \leq \frac{1}{\alpha} E_{\varepsilon_{0}}(0)
$$

Let us introduce the function

$$
\varphi(t):=\frac{1}{2}|u(t)|^{2} .
$$

Computations similar to those in the proof of Theorem 2.1 yield

$$
\ddot{\varphi}(t)+\alpha \dot{\varphi}(t)+2 E_{\varepsilon(t)}(t)=2\left|\frac{\mathrm{d} u}{\mathrm{~d} t}(t)\right|^{2},
$$

and it follows by the same method that

$$
\varphi(t)+\frac{2}{\alpha^{2}}\left(\mathrm{e}^{-\alpha t}-1+\alpha t\right) E_{\varepsilon(t)}(t) \leq \varphi(0)+\frac{1}{\alpha}\left(1-\mathrm{e}^{-\alpha t}\right) \dot{\varphi}(0)+2 h(t)
$$

where $h(t)$ is a bounded function. Hence $E_{\varepsilon(t)}(t) \leq C / t$ for some constant $C \geq 0$, and therefore

$$
\lim _{t \rightarrow \infty}\left|\frac{\mathrm{d} u}{\mathrm{~d} t}(t)\right|=\lim _{t \rightarrow \infty} a(u(t), u(t))=\lim _{t \rightarrow \infty} \varepsilon(t)|u(t)|^{2}=\lim _{t \rightarrow \infty} E_{\varepsilon(t)}(t)=0 .
$$


Furthermore, it follows that $\varphi(t)$ is bounded, which means that $u(t)$ is bounded in $H$. We claim that $\lim _{t \rightarrow \infty}|u(t)|$ exists. Indeed, since $E_{\varepsilon(t)}(t) \geq 0$, it follows from (10) that

$$
\ddot{\varphi}(t)+\alpha \dot{\varphi}(t) \leq 2\left|\frac{\mathrm{d} u}{\mathrm{~d} t}(t)\right|^{2},
$$

and we can apply Lemma 2.2 with $p(t)=2\left|\frac{\mathrm{d} u}{\mathrm{~d} t}(t)\right|^{2}$ to conclude that $\varphi(t)$ converges as $t \rightarrow \infty$. On the other hand, since $2 E_{\varepsilon(t)}(t) \geq \varepsilon(t)|u(t)|^{2}$, it follows that

$$
\ddot{\varphi}(t)+\alpha \dot{\varphi}(t)+\varepsilon(t)|u(t)|^{2} \leq 2\left|\frac{\mathrm{d} u}{\mathrm{~d} t}(t)\right|^{2} .
$$

Hence

$$
\dot{\varphi}(t)+\alpha \varphi(t)+\int_{0}^{t} \varepsilon(\tau)|u(\tau)|^{2} \mathrm{~d} \tau \leq \dot{\varphi}(0)+\alpha \varphi(0)+2 \int_{0}^{t}\left|\frac{\mathrm{d} u}{\mathrm{~d} t}(\tau)\right|^{2} \mathrm{~d} \tau .
$$

Since $\varphi(t) \geq 0$ and $|\dot{\varphi}(t)|=\left|\left(\frac{\mathrm{d} u}{\mathrm{~d} t}(t), u(t)\right)\right| \leq|\mathrm{d} u(t)||u(t)| \rightarrow 0$ as $t \rightarrow \infty$, we deduce that

$$
\int_{0}^{\infty} \varepsilon(\tau)|u(\tau)|^{2} \mathrm{~d} \tau \leq \dot{\varphi}(0)+\alpha \varphi(0)+2 \int_{0}^{\infty}\left|\frac{\mathrm{d} u}{\mathrm{~d} t}(\tau)\right|^{2} \mathrm{~d} \tau<\infty
$$

which would contradict (9) if $\lim _{t \rightarrow \infty}|u(t)|$ were a strictly positive number. Therefore $\lim _{t \rightarrow \infty}|u(t)|=0$, which completes the proof of the theorem.

Remark 2.5. Condition (9) means that $\varepsilon(t)$ decays slowly enough in order to let every trajectory converge to the stationary solution $u \equiv 0$. On the other hand, when $\varepsilon(t)$ tends to 0 fast enough, i.e. when $\int_{0}^{\infty} \varepsilon(\tau) \mathrm{d} \tau<\infty$, one can adapt to this situation the proof of the autonomous case and the limit solution $u_{\infty}$ may be different from 0. Similar results have been obtained for non-autonomous first-order in time equations governed by (sub)gradients of convex functions; in this direction, see [2] for slow-decay results and [12] for fast-decay ones. We refer the reader to [3] for similar results in the case of some second-order in time equations.

\section{Monotone CONSERVATIVE NONLINEARITY}

We are going now to extend the results of the previous section to a class of non-linear second-order in time equations. More precisely, we consider the infinite dimensional dynamical system generated by a non-linear equation of the type:

$$
\frac{\mathrm{d}^{2} u}{\mathrm{~d} t^{2}}(t)+\alpha \frac{\mathrm{d} u}{\mathrm{~d} t}(t)+A u(t)+f(u(t))=0, t>0
$$

with initial conditions

$$
u(0)=u_{0}, \quad \frac{\mathrm{d} u}{\mathrm{~d} t}(0)=v_{0},
$$

where $\alpha>0, u_{0} \in V, v_{0} \in H$, and $f: V \rightarrow H$ is continuous and monotone in a sense to be made precise. The hypotheses concerning the spaces $V, H$ and the linear operator $A$ are those of Section 2. On the non-linearity $f$, we first suppose that it is conservative, i.e.

$\left(\mathrm{h}_{4}\right) \exists F \in C^{1}(V ; \mathbb{R})$ such that $\left\langle F^{\prime}(u), v\right\rangle_{V^{\prime}, V}=(f(u), v)$.

Furthermore, we assume that $F$ is convex, which is equivalent to the monotonicity condition:

$\left(\mathrm{h}_{5}\right) \forall u, v \in V,(f(u)-f(v), u-v) \geq 0$. 
Therefore, the function $\Phi: V \rightarrow \mathbb{R}$ defined by

$$
\Phi(v):=\frac{1}{2} a(v, v)+F(v)
$$

satisfies $\Phi \in C^{1}(V ; \mathbb{R})$ with $\left\langle\Phi^{\prime}(u), v\right\rangle_{V^{\prime}, V}=a(u, v)+(f(u), v)$. Moreover, $\Phi$ is convex, which amounts to

$$
\forall u, v \in V, \quad a(u, v-u)+(f(u), v-u) \leq \Phi(v)-\Phi(u) .
$$

Thus, $\tilde{v} \in V$ satisfies $A \tilde{v}+f(\tilde{v})=0$ if and only if $\Phi(\tilde{v})=\min \{\Phi(v): v \in V\}$. Notice that under these conditions the set of equilibrium points $\{v \in V: A v+f(v)=0\}$ is a convex subset of $V$.

We say that the initial-value problem $(11,12)$ is well-posed when for every $u_{0} \in V$ and $v_{0} \in H$, there exists a unique function $u(\cdot) \in C\left(\left[0, \infty[; V) \cap C^{1}([0, \infty[; H)\right.\right.$ which satisfies (12), verifies (11) in the weak sense, and the corresponding energy function

$$
E(t):=\frac{1}{2}\left|\frac{\mathrm{d} u}{\mathrm{~d} t}(t)\right|^{2}+\Phi(u(t))
$$

is absolutely continuous with $\frac{\mathrm{d}}{\mathrm{d} t}[E(t)] \leq-\alpha\left|\frac{\mathrm{d} u}{\mathrm{~d} t}(t)\right|^{2}$ for a.e. $t>0$. It is not our purpose to develop the well-posedness of $(11,12)$ here, we only mention that some general sufficient conditions which guarantee this property are given in $[13,16,22]$.

Theorem 3.1. Assume that $\left(\mathrm{h}_{1}-\mathrm{h}_{5}\right)$ hold. Let $\alpha>0$ and suppose that the initial-value problem (11, 12) is well-posed. Given $u_{0} \in V$ and $v_{0} \in H$, let $u(\cdot) \in C\left(\left[0, \infty[; V) \cap C^{1}([0, \infty[; H)\right.\right.$ be the corresponding solution of $(11,12)$. If $S:=\{v \in V: A v+f(v)=0\}$ is nonempty then:

(i) $\frac{\mathrm{d} u}{\mathrm{~d} t} \in L^{2}(0, \infty ; H)$ and there exists $C \geq 0$ such that $E(t)-\min \Phi \leq C / t$. In particular, $\lim _{t \rightarrow \infty}\left|\frac{\mathrm{d} u}{\mathrm{~d} t}(t)\right|=0$ and $\lim _{t \rightarrow \infty} \Phi(u(t))=\min \Phi$;

(ii) there exists $u_{\infty} \in S$ such that $u(t) \rightarrow u_{\infty}$ weakly in $V$ as $t \rightarrow \infty$. Furthermore, $u(t) \rightarrow u_{\infty}$ strongly in $V$ iff $u(t) \rightarrow u_{\infty}$ strongly in $H$.

Proof. The proof of (i) is analogous to the linear case so that we only outline the main arguments. From $\frac{\mathrm{d}}{\mathrm{d} t}[E(t)] \leq-\alpha\left|\frac{\mathrm{d} u}{\mathrm{~d} t}(t)\right|^{2}$, it follows that

$$
\int_{0}^{\infty}\left|\frac{\mathrm{d} u}{\mathrm{~d} t}(\tau)\right|^{2} \mathrm{~d} \tau \leq \frac{1}{\alpha}[E(0)-\min \Phi]<\infty .
$$

Let $\tilde{v} \in S$ and define

$$
\varphi(t):=\frac{1}{2}|u(t)-\tilde{v}|^{2}
$$

We have

$$
\ddot{\varphi}(t)+\alpha \dot{\varphi}(t)=a(u(t), \tilde{v}-u(t))+(f(u(t)), \tilde{v}-u(t))+\left|\frac{\mathrm{d} u}{\mathrm{~d} t}(t)\right|^{2},
$$

which together with (13) yield

$$
\ddot{\varphi}(t)+\alpha \dot{\varphi}(t) \leq \Phi(\tilde{v})-\Phi(u(t))+\left|\frac{\mathrm{d} u}{\mathrm{~d} t}(t)\right|^{2} .
$$

Proceeding analogously to the linear case, we rewrite this inequality as

$$
\ddot{\varphi}(t)+\alpha \dot{\varphi}(t)+E(t) \leq \Phi(\tilde{v})+\frac{3}{2}\left|\frac{\mathrm{d} u}{\mathrm{~d} t}(t)\right|^{2},
$$


and, by computations similar to those used in the previous sections, we deduce that

$$
\varphi(t)+\frac{1}{\alpha^{2}}\left(\mathrm{e}^{-\alpha t}-1+\alpha t\right) E(t) \leq c_{0}+\frac{1}{\alpha^{2}}\left(\mathrm{e}^{-\alpha t}-1+\alpha t\right) \Phi(\tilde{v})+h(t)
$$

where $c_{0}:=\varphi(0)+\frac{1}{\alpha}\left(1-\mathrm{e}^{-\alpha t}\right) \dot{\varphi}(0)$ and $h(t)$ is an appropriate bounded function. In particular, since $\varphi(t) \geq 0$ and $\Phi(\tilde{v})=\min \Phi$, we have

$$
E(t) \leq \min \Phi+\frac{\alpha^{2}\left(c_{0}+h(t)\right)}{\mathrm{e}^{-\alpha t}-1+\alpha t}
$$

Letting $t \rightarrow \infty$, we get

$$
\limsup _{t \rightarrow \infty} \Phi(u(t)) \leq \limsup _{t \rightarrow \infty}\left[\frac{1}{2}\left|\frac{\mathrm{d} u}{\mathrm{~d} t}(t)\right|^{2}+\Phi(u(t))\right]=\limsup _{t \rightarrow \infty} E(t) \leq \min \Phi
$$

which suffices to ensure that $\lim _{t \rightarrow \infty} E(t)=\lim _{t \rightarrow \infty} \Phi(u(t))=\min \Phi$ and, consequently, $\lim _{t \rightarrow \infty}\left|\frac{\mathrm{d} u}{\mathrm{~d} t}(t)\right|=0$.

Let us turn to the proof of (ii). From $(14)$ and $\Phi(u(t)) \geq \Phi(\tilde{v})=\min \Phi$, it follows that

$$
\ddot{\varphi}(t)+\alpha \dot{\varphi}(t) \leq\left|\frac{\mathrm{d} u}{\mathrm{~d} t}(t)\right|^{2}
$$

and, by Lemma 2.2, $\varphi(t)$ converges as $t \rightarrow \infty$. In particular, $\{u(t)\}$ is bounded in $H$. On the other hand, since $F$ is convex, we have $F(u) \geq-b|u|-c$ for some constants $b, c \in \mathbb{R}$. Thus, from the estimate $\frac{1}{2} a(u, u) \leq \Phi(u)+b|u|+c$ we deduce that $a(u(t), u(t))$ is bounded, hence that $\{u(t)\}$ is bounded in $V$ thanks to $\left(\mathrm{h}_{2}\right)$. Let $\widehat{u} \in V$ be a cluster point of $\{u(t): t \rightarrow \infty\}$ for the weak topology of $V$. We have $u\left(t_{k}\right) \rightarrow \widehat{u}$ weakly in $V$ for some sequence $t_{k} \rightarrow \infty$ and, by the weak lower semi-continuity of $\Phi$, we obtain

$$
\Phi(\widehat{u}) \leq \liminf _{k \rightarrow \infty} \Phi\left(u\left(t_{k}\right)\right)=\lim _{t \rightarrow \infty} \Phi(u(t))=\min \Phi
$$

Therefore $\Phi(\widehat{u})=\min \Phi$, which amounts to $\widehat{u} \in S$. If $S$ is a singleton then $\{u(t): t \rightarrow \infty\}$ admits a unique cluster point for the weak topology in $V$, hence $u(t)$ converges weakly as $t \rightarrow \infty$. Otherwise, we apply the following argument due to Opial [19]. Let $\widehat{u}_{1}, \widehat{u}_{2} \in S$ be two cluster points of $\{u(t): t \rightarrow \infty\}$ for the weak topology of $V$. Recall that we have shown that for every $\tilde{v} \in S$, the corresponding $\varphi(t)=\frac{1}{2}|u(t)-\tilde{v}|^{2}$ converges as $t \rightarrow \infty$. In particular, $l_{i}:=\lim _{t \rightarrow \infty}\left|u(t)-\widehat{u}_{i}\right|^{2}$ exists for each $i=1,2$. Take a sequence $t_{k} \rightarrow \infty$ such that $u\left(t_{k}\right) \rightarrow \widehat{u}_{1}$ weakly in $V$. Since the injection from $V$ into $H$ is continuous, $u\left(t_{k}\right) \rightarrow \widehat{u}_{1}$ weakly in $H$. From the identity

$$
\left|u(t)-\widehat{u}_{1}\right|^{2}-\left|u(t)-\widehat{u}_{2}\right|^{2}=\left|\widehat{u}_{1}-\widehat{u}_{2}\right|^{2}+2\left(\widehat{u}_{1}-\widehat{u}_{2}, \widehat{u}_{2}-u(t)\right)
$$

we deduce that $l_{1}-l_{2}=-\left|\widehat{u}_{1}-\widehat{u}_{2}\right|^{2}$. Similarly, if we take $t_{j} \rightarrow \infty$ such that $u\left(t_{j}\right) \rightarrow \widehat{u}_{2}$ then $l_{1}-l_{2}=\left|\widehat{u}_{1}-\widehat{u}_{2}\right|^{2}$. Consequently, $\left|\widehat{u}_{1}-\widehat{u}_{2}\right|=0$. This establishes the uniqueness of the cluster point of $\{u(t): t \rightarrow \infty\}$ for the weak topology of $V$. Hence $u(t) \rightarrow u_{\infty}$ weakly in $V$ as $t \rightarrow \infty$ for some $u_{\infty} \in S$.

It remains to prove that if $u(t) \rightarrow u_{\infty}$ strongly in $H$ then $u(t) \rightarrow u_{\infty}$ strongly in $V$. By $\left(\mathrm{h}_{2}\right)$, we have

$$
\mu\left\|u(t)-u_{\infty}\right\|^{2} \leq \lambda\left|u(t)-u_{\infty}\right|^{2}+a\left(u(t)-u_{\infty}, u(t)-u_{\infty}\right)
$$

which we rewrite

$$
\frac{\mu}{2}\left\|u(t)-u_{\infty}\right\|^{2} \leq \frac{\lambda}{2}\left|u(t)-u_{\infty}\right|^{2}+\Phi(u(t))+\frac{1}{2} a\left(u_{\infty}, u_{\infty}\right)-a\left(u(t), u_{\infty}\right)-F(u(t)) .
$$


Since $u(t) \rightarrow u_{\infty}$ weakly in $V$, we have $\lim _{t \rightarrow \infty} a\left(u(t), u_{\infty}\right)=a\left(u_{\infty}, u_{\infty}\right)$. On the other hand, by weak lower semicontinuity of the continuous convex function $F: V \rightarrow \mathbb{R}$, we deduce that $\liminf _{t \rightarrow \infty} F(u(t)) \geq F\left(u_{\infty}\right)$. Therefore

$$
\limsup _{t \rightarrow \infty}\left[\frac{1}{2} a\left(u_{\infty}, u_{\infty}\right)-a\left(u(t), u_{\infty}\right)-F(u(t))\right] \leq-\frac{1}{2} a\left(u_{\infty}, u_{\infty}\right)-F\left(u_{\infty}\right)=-\Phi\left(u_{\infty}\right) .
$$

Recalling that $\lim _{t \rightarrow \infty} \Phi(u(t))=\min \Phi$ and $\Phi\left(u_{\infty}\right)=\min \Phi\left(\right.$ since $\left.u_{\infty} \in S\right)$, we obtain

$$
\limsup _{t \rightarrow \infty}\left\|u(t)-u_{\infty}\right\|^{2} \leq \frac{\lambda}{\mu} \lim _{t \rightarrow \infty}\left|u(t)-u_{\infty}\right|^{2},
$$

which completes the proof of the theorem.

Remark 3.2. Theorem 3.1 ensures weak convergence in $V$ under no compactness condition. Of course, as a consequence of this result, we have that if the trajectory $\{u(t): t \rightarrow \infty\}$ is precompact for the strong topology of $V$, then $u(t) \rightarrow u_{\infty} \in S$ strongly in $V$. Furthermore, according to Theorem 3.1(ii), in order to ensure strong convergence in $V$, we need only require the trajectory to be precompact for the strong topology of $H$. This is immediate when the injection from $V$ into $H$ is compact because $u(t) \rightarrow u_{\infty}$ weakly in $V$. Another interesting situation ensuring strong convergence in $V$ is the case where the non-linearity is such that the following symmetry property holds:

$$
\forall v \in V, F(-v)=F(v) .
$$

Indeed, by a simple adaptation of the arguments in the last part of the proof of Theorem 2.1, where it is considered the auxiliary function $q(t):=|u(t)|^{2}-\left|u\left(t_{0}\right)\right|^{2}-\frac{1}{2}\left|u(t)-u\left(t_{0}\right)\right|^{2}$ with $t \in\left[0, t_{0}\right]$, it is possible to prove that the convergence of the trajectory holds for the strong topology in $H$ even when the injection from $V$ into $H$ is not compact; we leave the details to the reader. One question still unanswered is whether strong convergence holds in the general setting of Theorem 3.1 without any additional condition.

We now turn to the stabilization of a particular equilibrium in this abstract non-linear setting and under a slow-decay condition. In addition to $\left(\mathrm{h}_{1}-\mathrm{h}_{5}\right)$, let us suppose that

$\left(\mathrm{h}_{6}\right) f(0)=0$.

Observe that under this hypothesis we have

$$
S:=\{v \in V: A v+f(v)=0\}=\operatorname{ker} A \cap\{v \in V: f(v)=0\} .
$$

Indeed, if $\tilde{v} \in S$ then $(A \tilde{v}, \tilde{v})+(f(\tilde{v}), \tilde{v})=0$. But by $\left(\mathrm{h}_{5}\right)$ together with $\left(\mathrm{h}_{6}\right)$, we have $(f(v), v) \geq 0$ for all $v \in V$; hence $(A \tilde{v}, \tilde{v})=(f(\tilde{v}), \tilde{v})=0$ and therefore $A \tilde{v}=0$.

Theorem 3.3. Let us assume that $\left(\mathrm{h}_{1}-\mathrm{h}_{6}\right)$ hold and let $u \in C\left(\left[0, \infty[; V) \cap C^{1}([0, \infty[; H)\right.\right.$ be a solution of

$$
\frac{\mathrm{d}^{2} u}{\mathrm{~d} t^{2}}(t)+\alpha \frac{\mathrm{d} u}{\mathrm{~d} t}(t)+A u(t)+f(u(t))+\varepsilon(t) u(t)=0, t>0,
$$

where $\alpha>0$ and $\varepsilon:[0, \infty[\rightarrow[0, \infty[$ is a given differentiable function such that for all $t \geq 0, \dot{\varepsilon}(t) \leq 0$. Suppose that the energy

$$
E_{\varepsilon(t)}(t):=\frac{1}{2}\left|\frac{\mathrm{d} u}{\mathrm{~d} t}(t)\right|^{2}+\frac{1}{2} a(u(t), u(t))+F(u(t))+\frac{\varepsilon(t)}{2}|u(t)|^{2}
$$

is absolutely continuous with $\frac{\mathrm{d}}{\mathrm{d} t}[E(t)] \leq-\alpha\left|\frac{\mathrm{d} u}{\mathrm{~d} t}(t)\right|^{2}+\frac{\dot{\varepsilon}(t)}{2}|u(t)|^{2}$ for a.e. $t>0$. Under these conditions, we have $\frac{\mathrm{d} u}{\mathrm{~d} t} \in L^{2}(0, \infty ; H)$, there exists $C \geq 0$ such that $E_{\varepsilon(t)}(t) \leq C / t, \frac{\mathrm{d} u}{\mathrm{~d} t}(t) \rightarrow 0$ strongly in $H$ and if $\int_{0}^{\infty} \varepsilon(\tau) \mathrm{d} \tau=\infty$ then $u(t) \rightarrow 0$ strongly in $V$ as $t \rightarrow \infty$. 
Proof. We only give the main ideas of the proof. From $\frac{\mathrm{d}}{\mathrm{d} t}[E(t)] \leq-\alpha\left|\frac{\mathrm{d} u}{\mathrm{~d} t}(t)\right|^{2}+\frac{\dot{\varepsilon}(t)}{2}|u(t)|^{2}$, it follows that $E_{\varepsilon(t)}(t)$ is non-increasing and

Setting $\varphi(t):=\frac{1}{2}|u(t)|^{2}$, we obtain

$$
\int_{0}^{\infty}\left|\frac{\mathrm{d} u}{\mathrm{~d} t}(\tau)\right|^{2} \mathrm{~d} \tau \leq \frac{1}{\alpha} E_{\varepsilon_{0}}(0)
$$

$$
\ddot{\varphi}(t)+\alpha \dot{\varphi}(t)=-a(u(t), u(t))-(f(u(t)), u(t))-\varepsilon(t)|u(t)|^{2}+\left|\frac{\mathrm{d} u}{\mathrm{~d} t}(t)\right|^{2} .
$$

Since for each $t>0$ the function $\Phi_{\varepsilon(t)}: V \rightarrow \mathbb{R}$ defined by

$$
\Phi_{\varepsilon(t)}(v):=\frac{1}{2} a(v, v)+F(v)+\frac{\varepsilon(t)}{2}|v|^{2}
$$

is convex and $\Phi_{\varepsilon(t)}^{\prime}(v)=A v+f(v)+\varepsilon(t) v$, we have in particular that for every $v \in V$

$$
\Phi_{\varepsilon(t)}(v)+(A v+f(v)+\varepsilon(t) v, 0-v) \leq \Phi_{\varepsilon(t)}(0)=F(0) .
$$

Observe that $\Phi_{\varepsilon(t)}^{\prime}(0)=0$ so that $F(0)=\Phi_{\varepsilon(t)}(0)=\min _{v \in V} \Phi_{\varepsilon(t)}(v)$. Without loss of generality we can assume that $F(0)=0$. Therefore

which may be rewritten

$$
\ddot{\varphi}(t)+\alpha \dot{\varphi}(t)+\Phi_{\varepsilon(t)}(u(t)) \leq\left|\frac{\mathrm{d} u}{\mathrm{~d} t}(t)\right|^{2},
$$

The standard integration procedure yields

$$
\ddot{\varphi}(t)+\alpha \dot{\varphi}(t)+E_{\varepsilon(t)}(t) \leq \frac{3}{2}\left|\frac{\mathrm{d} u}{\mathrm{~d} t}(t)\right|^{2} .
$$

$$
\varphi(t)+\frac{2}{\alpha^{2}}\left(\mathrm{e}^{-\alpha t}-1+\alpha t\right) E_{\varepsilon(t)}(t) \leq \varphi(0)+\frac{1}{\alpha}\left(1-\mathrm{e}^{-\alpha t}\right) \dot{\varphi}(0)+h(t),
$$

where $h(t)$ is a bounded function. Hence $E_{\varepsilon(t)}(t) \leq C / t$ for some constant $C \geq 0$ and, since $E_{\varepsilon(t)}(t) \geq$ 0, we deduce that $\lim _{t \rightarrow \infty}\left|\frac{\mathrm{d} u}{\mathrm{~d} t}(t)\right|=\lim _{t \rightarrow \infty} a(u(t), u(t))=\lim _{t \rightarrow \infty} \varepsilon(t)|u(t)|^{2}=\lim _{t \rightarrow \infty} \Phi_{\varepsilon(t)}(u(t))=\lim _{t \rightarrow \infty} E_{\varepsilon(t)}(t)=0$. Furthermore, $\varphi(t)$ converges as $t \rightarrow \infty$ by Lemma 2.2. We leave it to the reader to verify that if $\lim _{t \rightarrow \infty} \varphi(t)$ were strictly positive, then this would yield a contradiction. Thus $\lim _{t \rightarrow \infty}|u(t)|=\lim _{t \rightarrow \infty} a(u(t), u(t))=0$, which gives $\lim _{t \rightarrow \infty}\|u(t)\|=0$ thanks to $\left(\mathrm{h}_{2}\right)$.

\section{Some EXAmples And COMments}

Let $\Omega \subset \mathbb{R}^{N}$ be an open bounded set with boundary $\partial \Omega$ sufficiently regular. Let us consider the equation

$$
\left.u_{t t}+\alpha u_{t}-\Delta u+f(u)=0 \quad \text { in } \quad \Omega \times\right] 0, \infty[
$$

where $\alpha>0$ and $f \in C^{2}(\mathbb{R} ; \mathbb{R})$. This equation is supplemented with the Neumann boundary condition

$$
\left.\frac{\partial u}{\partial \mathbf{n}}=0 \quad \text { on } \quad \partial \Omega \times\right] 0, \infty[.
$$

The functional setting of the evolution problem $(15,16)$ is given by $H=L^{2}(\Omega), V=H^{1}(\Omega)$ and $a(u, v)=$ $\int_{\Omega} \nabla u(x) \cdot \nabla v(x) \mathrm{d} x$ so that $A=-\Delta$ in $H^{1}(\Omega)$. Define $F(s):=\int_{0}^{s} f(r) \mathrm{d} r$ and assume that $\lim \inf _{|s| \rightarrow \infty} \frac{F(s)}{s^{2}}>0$, 
and that there exists $c_{1}>0$ such that $\liminf _{|s| \rightarrow \infty} \frac{s f(s)-c_{1} F(s)}{s^{2}}>0$. We also suppose that $\left|f^{\prime}(s)\right| \leq c_{2}\left(1+|s|^{\gamma}\right)$ with $0 \leq \gamma<\infty$ when $N=1,2,0 \leq \gamma \leq 2$ when $N=3$, and $\gamma=0$ when $N \geq 4$. For simplicity of notation, we write $F(u)$ instead of $\int_{\Omega} F(u(x)) \mathrm{d} x$. The well-posedness of this initial-value problem is a consequence of general results from dynamical systems theory applied to the equivalent equation $u_{t t}+\alpha u_{t}-\Delta u+\eta u+f_{\eta}(u)=0$, where $f_{\eta}(s)=-\eta s+f(s)$ and $\eta>0$ is small enough, and, moreover $\{u(t): t \rightarrow \infty\}$ is precompact in $H^{1}(\Omega)$; we refer the reader to [22] (Chap. IV, Ex. 4.1). Furthermore, we assume that $f$ is nondecreasing, which ensures the monotonicity condition $\left(\mathrm{h}_{5}\right)$. By Theorem 3.1 and Remark 3.2, every solution of $(15,16)$ strongly converges in $H^{1}(\Omega)$, as $t \rightarrow \infty$, toward a solution of

$$
\left\{\begin{aligned}
-\Delta u+f(u) & =0 \text { in } \Omega \\
\frac{\partial u}{\partial \mathbf{n}} & =0 \text { on } \partial \Omega
\end{aligned}\right.
$$

A similar convergence result is valid for the evolution problem:

$$
\left\{\begin{aligned}
u_{t t}+\alpha u_{t}-\Delta u-\lambda_{1} u+f(u) & =0 & & \text { in } \Omega \times] 0, \infty[, \\
u & =0 & & \text { on } \partial \Omega \times] 0, \infty[
\end{aligned}\right.
$$

where $\lambda_{1}$ is the first eigenvalue of $-\Delta$ in $H_{0}^{1}(\Omega)$. In this case, we take $H=L^{2}(\Omega), V=H_{0}^{1}(\Omega)$ and $a(u, v)=$ $\int_{\Omega}\left[\nabla u(x) \cdot \nabla v(x)-\lambda_{1} u(x) v(x)\right] \mathrm{d} x$ so that $A=-\Delta-\lambda_{1} I$.

Observe that in both examples, the kernel of $A$ is one-dimensional so that the set of equilibria can be identified with an interval of $\mathbb{R}$. This situation has already been studied in $[15,23]$ for a more general quasi-linear and monotone damping term $g\left(u_{t}\right)$, obtaining some convergence results by a different method relying on topological arguments. But the dimension of $A$ plays no role in our approach and this allows us to consider other situations. For instance, $-\Delta$ in $H^{1}(\Omega)$ can be replaced by the bi-Laplacian operator $\Delta^{2}$ in $H^{2}(\Omega)$, and $-\Delta-\lambda_{1} I$ in $H_{0}^{1}(\Omega)$ can be replaced by $\Delta^{2}-\mu_{1} I$ where $\mu_{1}$ is the first eigenvalue of $\Delta^{2}$ in $H_{0}^{2}(\Omega)$, no matter whether $\mu_{1}$ is simple or not (see [9] for an example where $\mu_{1}$ is double). Nevertheless, the convergence of the trajectories in theses cases is a consequence of some general results of Hale and Raugel [14], and Brunovsky and Polacik [8]. Roughly speaking, it follows from the results in [8] that if the linearized dynamical system at some point $z$ in the $\omega$ limit set corresponding to an initial data $y_{0}$ admits 1 as eigenvalue of multiplicity $m$ and $z$ is contained in a sub-manifold $M$ of equilibria of dimension $m$, then the whole orbit of $y_{0}$ converges to $z$ (the case $m=1$ was treated in [14]). In the case of wave-like equations, this result allows the kernel of the linearized operator at $z=(u, 0)$ to have a dimension higher than one and, moreover, monotonicity of $f$ is not required. On the other hand, we exploit global monotonicity to establish convergence by energy and functional methods, which are more elementary than those used in $[8,14]$.

Another feature of our approach is that it does not require as assumptions neither coercivity of $F$ nor precompactness conditions on the trajectory (see Rem. 3.2). The latter is interesting in the case where the physical domain $\Omega$ is not bounded. Indeed, the compactness of the injection between the corresponding function spaces $V$ and $H$ is lost for unbounded domains so that compactness properties of the associated semi-group are difficult to obtain. This is a major inconvenient for the applying of the standard methods of dynamical system theory. Without additional assumptions, our result applies under such lack of compactness but only ensures weak convergence in $V$.

Of course, other boundary conditions can be considered as spatial-periodicity, and the abstract results apply also for coupled systems of differential equations.

The authors wish to thank Prof. E. Zuazua and the anonymous referees for some comments which were very useful to improve the presentation of this paper. The authors are particularly grateful to the referee that indicated to them the reference Brunovsky-Polacik [8]. 


\section{REFERENCES}

[1] F. Alvarez, On the minimizing property of a second order dissipative system in Hilbert spaces. SIAM J. Control Optim. 38 (2000) 1102-1119.

[2] H. Attouch and R. Cominetti, A dynamical approach to convex minimization coupling approximation with the steepest descent method. J. Differential Equations 128 (1996) 519-540.

[3] H. Attouch and M.O. Czarnecki, Asymptotic control and stabilization of nonlinear oscillators with non-isolated equilibria. $J$. Differential Equations (to appear).

[4] H. Attouch, X. Goudou and P. Redont, A dynamical method for the global exploration of stationary points of a real-valued mapping: The heavy ball method. Communications in Contemporary Math. 2 (2000) 1-34.

[5] B. Aulbach, Approach to hyperbolic manifolds of stationary solutions. Springer-Verlag, Lecture Notes in Math. 1017 (1983) $56-66$.

[6] H. Brezis, Opérateurs maximaux monotones et semigroupes de contractions dans les espaces de Hilbert. North-Holland, Amsterdam, Math. Studies 5 (1973).

[7] R.E. Bruck, Asymptotic convergence of nonlinear contraction semigroups in Hilbert space. J. Funct. Anal. 18 (1975) 15-26.

[8] P. Brunovsky and P. Polacik, The Morse-Smale structure of a generic reaction-diffusion equation in higher space dimension. J. Differential Equations 135 (1997) 129-181.

[9] C.V. Coffman, R.J. Duffin and D.H. Shaffer, The fundamental mode of vibration of a clamped annular plate is not of one sign, Constructive Approaches to Math. Models. Academic Press, New York-London-Toronto, Ont. (1979) 267-277.

[10] C.M. Dafermos and M. Slemrod, Asymptotic behavior of nonlinear contraction semigroups. J. Funct. Anal. 13 (1973) 97-106.

[11] R. Dautray and J.-L. Lions, Analyse mathématique et calcul numérique, Vol. 8, Évolution : semi-groupe, variationnel. Masson, Paris (1988).

[12] H. Furuya, K. Miyashiba and N. Kenmochi, Asymptotic behavior of solutions to a class of nonlinear evolution equations. $J$. Differential Equations 62 (1986) 73-94.

[13] J.M. Ghidaglia and R. Temam, Attractors for damped nonlinear hyperbolic equations. J. Math. Pures Appl. 66 (1987) $273-319$.

[14] J. Hale and G. Raugel, Convergence in gradient-like systems with applications to PDE. Z. Angew. Math. Phys. 43 (1992) $63-124$.

[15] A. Haraux, Asymptotics for some nonlinear hyperbolic equations with a one-dimensional set of rest points. Bol. Soc. Brasil. Mat. 17 (1986) 51-65.

[16] A. Haraux, Semilinear Hyperbolic Problems in Bounded Domains, Mathematical Reports 3(1). Harwood Academic Publishers, Gordon and Breach, London (1987).

[17] A. Haraux and M.A. Jendoubi, Convergence of bounded weak solutions of the wave equation with dissipation and analytic nonlinearity. Calc. Var. Partial Differential Equations 9 (1999) 95-124.

[18] M.A. Jendoubi, Convergence of global and bounded solutions of the wave equation with linear dissipation and analytic nonlinearity. J. Differential Equations 144 (1998) 302-312.

[19] Z. Opial, Weak convergence of the sequence of successive approximations for nonexpansive mappings. Bull. Amer. Math. Soc. 73 (1967) 591-597.

[20] A. Pazy, On the asymptotic behavior of semigroups of nonlinear contractions in Hilbert space. J. Funct. Anal. 27 (1978) 292-307.

[21] L. Simon, Asymptotics for a class of non-linear evolution equations, with applications to geometric problems. Ann. Math. 118 (1983) 525-571.

[22] R. Temam, Infinite-Dimensional Dynamical Systems in Mechanics and Physics. Springer-Verlag, New York, Appl. Math. Sci. 68 (1988).

[23] E. Zuazua, Stability and decay for a class of nonlinear hyperbolic problems. Asymptot. Anal. 1 (1988) 161-185. 